Accurate Region Proposal Generation and Joint Object Detection // Proc of the IEEE Conference on Computer Vision and Pattern Recognition. Washington, USA : IEEE, 2016: 845-853.

[26] GIDARIS S, KOMODAKIS N. Object Detection via a Multi-region and Semantic Segmentation-Aware CNN Model // Proc of the IEEE International Conference on Computer Vision. Washington, USA : IEEE, 2015: 1134-1142.

\section{作者简介}

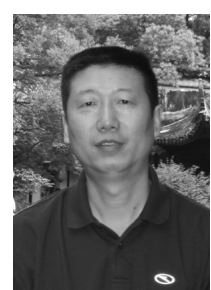

王 浩, 博士, 教授, 主要研究方向为智能计 算理论与软件、人工智能、机器视觉、数据 挖掘等.E-mail:jsjxwangh@ hfut. edu.cn.

(WANG Hao, Ph. D., professor. His research interests include intelligent computing theory and software, artificial intelligence, machine vision and data mining. )

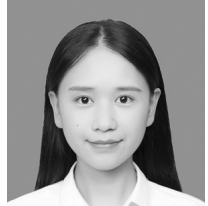

单文静, 硕士研究生, 主要研究方向为计算 机应用技术、目标检测、机器学习. E-mail: 13856937632@163.com.

(SHAN Wenjing, master student. Her research interests include computer application technology, object detection and machine learning. )

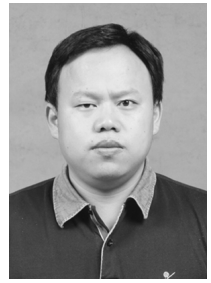

方宝富(通讯作者), 博士, 副教授, 主要研 究方向为机器学习、机器视觉、多机器人系 统.E-mail:fangbf@ hfut.edu.cn.

(FANG Baofu ( Corresponding author), Ph. D. , associate professor. His research interests include machine learning, machine vision and multi-robot systems. )

\title{
2020 年中国粒计算与知识发现学术会议 (CGCKD2020) 征文通知
}

由中国人工智能学会主办, 中国人工智能学会粒计算与知识发现专委会协办, 国际粗䊁集学会支持, 山 西大学承办的 2020 年中国粒计算与知识发现学术会议 (第二十届中国粗粘集与软计算学术会议、第十四届 中国粒计算学术会议、第八届三支决策学术会议)将于 2020 年 8 月 14 日至 16 日在山西省太原市召开。现 将会议有关征文事宜通知如下,热忱欢迎相关研究人员踊跃投稿并参会。

\section{一、征文范围(包括但不限于)}

1 、粗糙集与软计算

粗䊁集理论及应用、模糊集理论及应用、近似推理与不确定性分析、多准则决策分析、进化计算、神经计 算、类脑计算、认知计算、云模型理论及其应用等。

2、粒计算理论及其应用

商空间理论及应用、粒逻辑与推理、形式概念分析、信息粒的表示、信息粒化、粒度选择、粒计算的模式分 析与处理、多粒度推理模型、多粒度认知计算、多粒度智能决策、多粒度时空数据挖掘等。

3 、三支决策模型与分析

三支决策与粗䊁集、三支决策与区间集、三支决策与粒计算、三支决策与模糊集、三支决策与概念格、三 支决策的数学模型、三支决策空间、三支聚类、动态三支决策、序贯三支决策等。

4 、知识发现与数据挖掘

分类学习、数据聚类、关联和相关性、时间序列挖掘、知识演化、衰退分析、决策树、神经网络、遗传算法、 模糊分类和聚类、粗糙分类、规则归纳、多粒度深度学习、多粒度知识发现和建模、多尺度媒体数据分析等。

5 、人工智能创新应用 


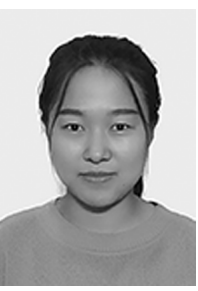

赵梦瑶, 博士研究生, 主要研究方向为视频 异常行为检测. E-mail: zhaomengyao0826@ 126. com.

(ZHAO Mengyao, Ph. D. candidate. Her research interests include video abnormal behavior detection. )

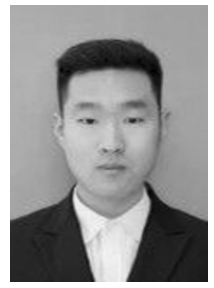

辛丙一, 硕士研究生, 主要研究方向为鲁棒 模式识别技术. E-mail: $1194326336 @ q q$. com.

(XIN Bingyi, master student. His research interests include robust pattern recognition technology. )

(上接 120 页)

\section{二、重要日期}

论文投稿截止日期 2020 年 4 月 15 日

论文录用通知日期 2020 年 6 月 10 日

论文修改与终稿提交日期 2020 年 6 月 25 日

\section{三、注意事项}

1、会议采用网上投稿方式,投稿地址: https://easychair. org/conferences/? conf $=$ cgckd2020

2、会议接收中英文论文。中文稿件请按照《计算机研究与发展》模版排版,英稿件请按照 IEEE 常规格 式排版, 原则上不超过 8000 字。会议将履行严格的审稿程序, 遵循宁缺勿滥的原则, 杜绝抄袭、数据不实、 一稿多投等学术不端行为。

3 、投稿内容应突出作者的创新与成果, 具有较重要的学术价值与应用推广价值, 且未在公开发行的刊物 上发表或会议上宣读。请在稿件最后附上通讯作者姓名、性别、职务/职称、所属单位、通信地址、邮政编码、 联系电话和 E-mail 地址。

4、根据论文评审情况, 所有录用论文将根据论文质量推荐到《International Journal of Machine Learning and Cybernetics》、《International Journal of Bio-inspired Computation》、《CAAI Transactions on Intelligence Technology》、《International Journal of Computer Science and Knowledge Engineering》、《计算机研究与发展》、《模式 识别与人工智能》、《计算机科学》、《计算机科学与探索》、《小型微型计算机系统》、《智能系统学报》、《南京 大学学报 (自然科学版)》、《计算机应用》、《计算机工程与应用》、《数据采集与处理》、《山东大学学报 (理学 版)》、《郑州大学学报 (理学版)》、《山西大学学报(自然科学版)》、《数码设计》等期刊正刊发表。在各期刊 发表前,作者须按照审稿意见和期刊要求修改论文;每篇录用论文至少有一名作者注册参会。

5、投稿时,请作者在论文首页左上角注明 “希望本论文被大会推荐至 xxx 期刊”,每一篇论文可以选择 $2 \sim 3$ 个期望被推荐的期刊。如果希望推荐到英文期刊,请用英文撰写。

6 、会议将评选优秀学生论文若干。候选的优秀学生论文第一作者必须是在校学生, 且必须由学生到会 宣讲。如希望参评,投稿时请注明第一作者是学生。

\section{四、联系方式}

投稿与会务咨询:

\section{魏巍 18635125988 翟岩慧 13100147204 钱宇华 15135193000}

会务邮箱 cgckd2020@163.com

通信地址 山西省太原市小店区坞城路 92 号山西大学计算机与信息技术学院 邮编 030006

会议网站 http://cgckd2020. sxu. edu. cn 